\title{
WAVELENGTH CALIBRATION OF BALLOON-BORNE ULTRAVIOLET \\ STELLAR SPECTROGRAPH
}

\author{
R. Hoekstra \\ Space Research Laboratory of the Astronomical Institute at Utrecht \\ the Netherlands
}

The procedure will be described that is developed for assigning wavelengths to the UV stellar spectra obtained in 1976 by means of a balloon-borne instrument consisting of telescope with starpointing system, echelle spectrograph and SEC vidicon. The instrument covers the spectral region of $2000 \AA$ to $3400 \AA$ with a wavelength resolution of $0.7 \AA$. The wavelength assignment aims at an accuracy of $0.01 \AA$. Results will be presented and difficulties encountered, related with the use of echelle spectrograph and SEC vidicon detector, will be anaiysed. 